\title{
Marangoni flow in floating half zone of molten tin
}

\author{
Kai $\mathrm{Li}^{\mathrm{a}, *}$, Satoshi Matsumoto ${ }^{\mathrm{b}}$, Nobuyuki Imaishi ${ }^{\mathrm{c}, *}$, Wen-Rui $\mathrm{Hu}^{\mathrm{a}}$ \\ ${ }^{a}$ Key Laboratory of Microgravity, Institute of Mechanics, Chinese Academy of Sciences, Beijing 100190, China \\ ${ }^{\mathrm{b}}$ Institute of Space and Astronautical Science, Japan Aerospace Exploration Agency, 2-1-1 Sengen, Tsukuba, Japan \\ ${ }^{\mathrm{c}}$ Institute for Materials Chemistry and Engineering, Kyushu University, 6-1 Kasuga Koen, Kasuga 816-8580, Japan
}

\section{A R T I C L E I N F O}

\section{Article history:}

Received 20 January 2014

Received in revised form 20 November 2014

Accepted 3 December 2014

Available online 8 January 2015

\section{Keywords:}

Marangoni flow

Flow instabilities

Deformed half zone

Buoyancy

\begin{abstract}
A B S T R A C T
Gravity affects the stability of Marangoni flow in floating half zone of low-Pr fluids through two different factors, i.e., the buoyancy and the static deformation of the free surface shape. In the present study, influence of these two factors are evaluated by unsteady three-dimensional (3D) simulations for a realistic model of floating half zone of molten tin $(\mathrm{Pr}=0.009)$ with an aspect ratio As $=2.0$ under a ramped temperature difference $(1.19 \mathrm{~K} / \mathrm{min})$ between the top and bottom ends of two iron supporting rods. The corresponding first critical conditions for the onset of 3D asymmetric non-oscillatory flows and the second critical conditions for the onset of 3D oscillatory flows are determined. Simulation results indicate that the fee surface deformation is the most influential factor for the critical conditions of the flow transitions. Buoyancy is less influential to the flow transitions. However, buoyancy causes multiple step transitions between different 3D asymmetric non-oscillatory flow modes.
\end{abstract}

(c) 2014 Elsevier Ltd. All rights reserved.

\section{Introduction}

The stability of the surface tension driven flow (Marangoni flow) in floating half zone of low-Pr fluids has been the subject of extensive research for decades. It was motivated by the experimental fact that an oscillatory Marangoni flow in floating zone configurations may be responsible for striations in crystals grown using a partially covered floating zone method [1] and floating zone Si crystal grown in space [2]. Since the first prediction through the three-dimensional (3D) numerical simulation by Rupp et al. [3], numerous linear stability analyses (LSA), e.g. [4-7], and nonlinear numerical simulations, e.g. $[5,6]$, have proved that Marangoni flow in floating half zone (noted as FHZ, hereafter) of low-Pr fluids becomes oscillatory through a two step bifurcations: the first bifurcation from an axisymmetric non-oscillatory flow to a 3D asymmetric non-oscillatory flow occurs at a certain Reynolds number (the first critical Reynolds number: $\mathrm{Re}_{\mathrm{c} 1}$ ) and the second bifurcation to a 3D oscillatory flow occurs at much larger Reynolds number (the second critical Reynolds number: $\mathrm{Re}_{\mathrm{c} 2}$ ). Moreover, these 3D flows in FHZ of low-Pr fluids are caused by hydrodynamic instability mechanism [4,5], rather than the hydrothermal wave instability mechanism [8] which is widely known as the cause of the oscillatory 3D flow in FHZs of moderate-Pr and high-Pr fluids

\footnotetext{
* Corresponding authors.

E-mail addresses: likai@imech.ac.cn (K. Li), imaishi@cm.kyushu-u.ac.jp (N. Imaishi).
}

[4,5]. Velten et al. [9] experimentally found that the critical Reynolds number for the onset of oscillatory flow in FHZ of moderate-Pr fluid heated from bottom is larger than that obtained when heated from top. Wanschura et al. [10] studied the effect of gravity on the Marangoni flow in FHZ by linear stability analysis and confirmed the counterintuitive experimental fact found by Velten et al. [9]. However, the mechanism was not clearly explained. Also their analysis predicts this apparently contradictory stabilizing effect of buoyancy may occur in FHZ of low-Pr fluid. These theoretical and numerical predictions are not yet validated by comparing with reliable experimental results. There are many experimental reports on the oscillation frequency and flow patterns of oscillatory flow in FHZ of low-Pr fluids such as molten silicon [11] and molten silver [12]. However, these high temperature experiments are unsuitable for measuring a small temperature difference to detect the critical conditions. There are some studies on oscillatory Marangoni flow in FHZ at lower temperatures, e.g. Han et al. with mercury [13] and Yang and Kou with molten tin [14]. According to these experiments, $\mathrm{Ma}_{\mathrm{c} 2}$ for a transition to oscillatory flow ranges between 200 and 900 and $\mathrm{Ma}_{\mathrm{c} 1}$ was not detected. JAXA started a series of on-ground experiments, e.g. [15-17], searching both flow transition conditions, $\operatorname{Re}_{\mathrm{c} 1}$ and $\mathrm{Re}_{\mathrm{c} 2}$ in $\mathrm{FHZ}$ of molten tin $(\operatorname{Pr}=0.009)$. Correspondingly, a series of numerical simulations on Marangoni flow in FHZ of molten tin have been conducted based on a simple FHZ model such as a cylindrical FHZ held between two circular heating/cooling disks $[18,19]$ or a cylindrical FHZ held between two iron rods $[20,21]$. In these simulations, effects of the aspect 
ratio of $\mathrm{FHZ}$ on the flow instability under microgravity condition were systematically studied. Li et al. [21] studied the effect of ramping rate of temperature difference by numerical simulations. It was confirmed that the larger temperature ramping retards the flow transitions which raised the experimentally determined $\mathrm{Re}_{\mathrm{c} 1}$ and $\operatorname{Re}_{\mathrm{c} 2}$. However, buoyancy and free surface deformation caused by the gravity were not considered in these studies.

For a quantitative comparison of the critical conditions determined through numerical simulations to the results through on-ground experiments, numerical simulations must include all gravity-related factors, i.e. static deformation of free surface and the buoyancy. Many numerical studies on flow instabilities have been done for non-cylindrical FHZ under microgravity, i.e., the effect of liquid volume. These works were reviewed and benchmark-tested by Shevtsova [22]. To our knowledge, there are only a few linear stability analyses and numerical simulations on the instabilities of buoyant-Marangoni convection in gravitationary deformed FHZ of low-Pr fluids. Nienheuser and Kuhlmann [23] conducted a linear stability analysis of axisymmetric Marangoni flow in FHZ with non-cylindrical surfaces for $\operatorname{Pr}=0.02$ and $\operatorname{Pr}=4$. Kuhlmann et al. [24] conducted linear stability analysis of axisymmetric flow in gravitationary deformed FHZ with unity aspect ratio for $\operatorname{Pr}=0 \sim 0.04$. In the papers, they provided a plot of $\mathrm{Re}_{\mathrm{c} 1}$ as a function of Bond number and heating direction for $\operatorname{Pr}=0.023$. Lappa et al. [25] studied stability limit of Marangoni flow in deformed $\mathrm{FHZ}$ of silicon $(\mathrm{Pr}=0.01)$ and gallium $(\mathrm{Pr}=0.04)$ by comparing with their own results for cylindrical FHZ with an aspect ratio As $=2.0$. These works treated the static deformation of the surface. Shevtsova [22] included $\mathrm{Re}_{\mathrm{c} 1}$ for gravitationary deformed $\mathrm{FHZ}$ in the benchmark topics but $\operatorname{Re}_{\mathrm{c} 2}$ was not tested. However, the combined effects of buoyancy and free surface deformation on the Marangoni flow in FHZ of low-Pr fluids under earth gravity $(1 G)$ are yet not fully discerned. It should be noted that Kuhlmann and Nienheuser et al. [23] studied the effect of dynamic surface deformation on the instability and the amplitudes of dynamic deformations are so small that would not cause significant effects.

In the present study, the combined effects of buoyancy and static surface deformation on time evolution of Marangoni flow and flow transition processes in a $\mathrm{FHZ}$ of $\mathrm{Pr}=0.009$ with $\mathrm{As}=2.0$ under a ramped temperature difference are studied by numerical simulations based on a realistic FHZ model. A ramp rate of $1.19 \mathrm{~K} / \mathrm{min}$ is assumed for the time-dependent temperature difference between both ends of the two supporting iron rods. In order to distinguish effects of free surface deformation and buoyancy, behavior of FHZ under special situations, i.e., cylindrical FHZ with buoyancy (Bo $=0, \mathrm{Gr} \neq 0$ ) and deformed $\mathrm{FHZ}$ without buoyancy $(B o \neq 0, G r=0)$ were also studied using two imaginary fluids. These imaginary fluids, Fluid A and Fluid B, are assumed to have the same thermophysical properties of molten tin except for either surface tension (quite large surface tension) or thermal expansion coefficient (no thermal expansion), respectively.

\section{Model and methods}

The schematics of a realistic FHZ of molten tin are shown in Fig. 1. The melt zone of molten tin is supported between two iron rods. The melt is assumed to be an incompressible Newtonian fluid and further adopted the Boussinesq assumption except for the temperature dependencies of density and surface tension. Thermophysical properties of molten tin and iron rod are shown in Table 1 together with the two imaginary fluids (Fluid A and Fluid B). A cylindrical coordinate system $(\mathrm{R}, \theta, \mathrm{Z})$ is adopted with the origin located at the center of the lower melt/rod interface. The radius of the supporting rod is denoted as $a$. The melt zone length and

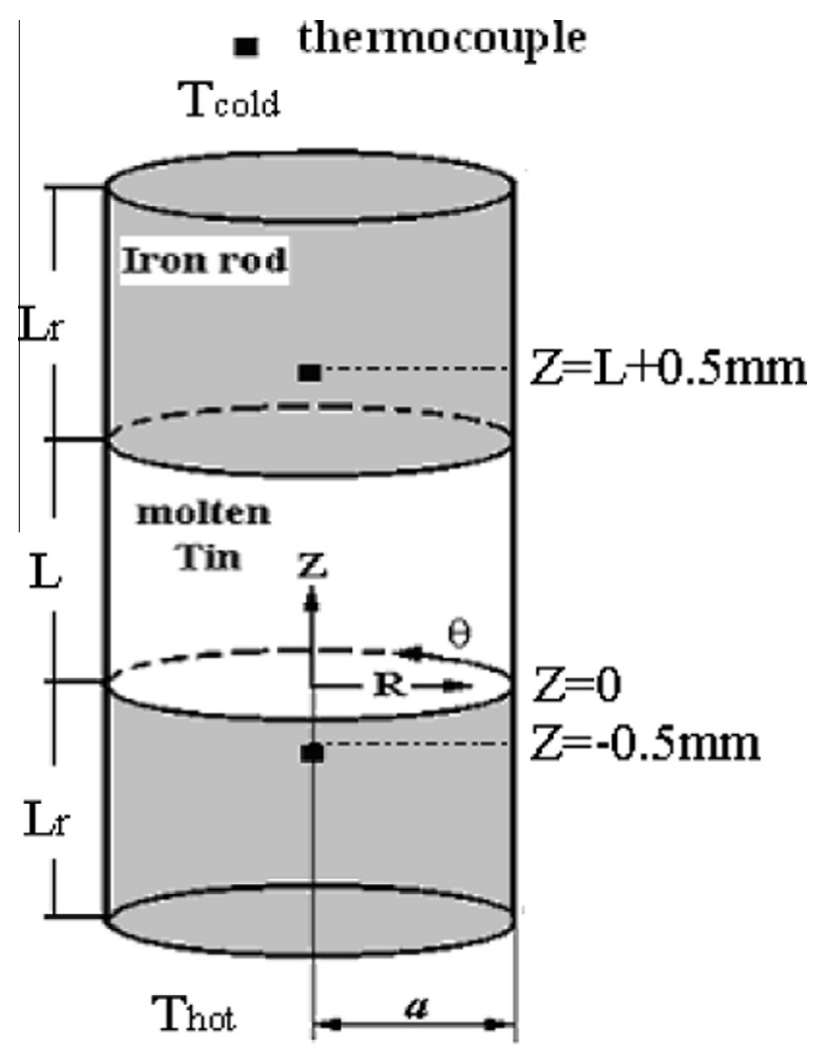

Fig. 1. Schematics of a realistic floating half zone (FHZ) model.

Table 1

Thermophysical properties.

\begin{tabular}{lllll}
\hline & Molten tin & Fluid A & Fluid B & Iron \\
\hline$\rho\left(\mathrm{kg} / \mathrm{m}^{3}\right)$ & 6793 & 6793 & 6793 & 7700 \\
$k\left(\mathrm{~W} / \mathrm{mK}^{2}\right)$ & 35.44 & 35.44 & 35.44 & 20.0 \\
$C_{p}(\mathrm{~J} / \mathrm{kgK})$ & 242 & 242 & 242 & 460 \\
$\nu\left(\mathrm{m}^{2} / \mathrm{s}\right)$ & $1.94 \times 10^{-7}$ & $1.94 \times 10^{-7}$ & $1.94 \times 10^{-7}$ & - \\
$\sigma(\mathrm{N} / \mathrm{m})$ & 0.5 & $>>1$ & 0.5 & - \\
$\sigma_{\mathrm{T}}(\mathrm{N} / \mathrm{mK})$ & $-1.3 \times 10^{-4}$ & $-1.3 \times 10^{-4}$ & $-1.3 \times 10^{-4}$ & - \\
$\beta(1 / \mathrm{K})$ & $1.3 \times 10^{-4}$ & $1.3 \times 10^{-4}$ & 0 & - \\
\hline
\end{tabular}

iron rod length are denoted $L$ and $L_{\mathrm{r}}$ with the aspect ratios As $=L / a$ and $A s_{\mathrm{r}}=L_{\mathrm{r}} / a$, respectively. In the present study, ( $a=3.0 \mathrm{~mm}, \mathrm{~A}=\mathrm{As}_{\mathrm{r}}=2.0$ ) is adopted. The fluid is initially motionless and kept at an uniform temperature $T_{0}=703.6 \mathrm{~K}$, and subsequently linearly increasing temperature difference were applied on the top end and bottom end of the supporting iron rods $\left(T_{\mathrm{H}}\right.$ and $\left.T_{\mathrm{C}}\right)$. Based on the experimental average ramping rate $0.34 \mathrm{~K} / \mathrm{min}$ measured through the thermocouples located at $0.5 \mathrm{~mm}$ apart from the melt/rod interfaces (see Fig. 1) during one of the experiments conducted at JAXA [15], the ramping rate of the temperature difference is determined as $\mathrm{d}\left(T_{\mathrm{H}}-T_{\mathrm{C}}\right) / \mathrm{d} t=1.19 \mathrm{~K} / \mathrm{min}$ by the pure steady conduction model $[19,20]$. Therefore, the time-dependent temperatures at the ends of the supporting rods are $T_{\mathrm{H}}=703.6+0.009917 t[\mathrm{~K}] \quad$ and $\quad T_{\mathrm{C}}=703.6-0.009917 t[\mathrm{~K}]$, respectively.

The fundamental equations are expressed as follows:

In the melt zone:

$\nabla \cdot \mathbf{U}=0$

$\frac{\partial \mathbf{U}}{\partial t}+\mathbf{U} \cdot \nabla \mathbf{U}=\frac{1}{\rho} \nabla p+v \nabla^{2} \mathbf{U}-\beta g\left(T-T_{0}\right) \mathbf{e}_{z}$ 
$\frac{\partial T}{\partial t}+\mathbf{U} \cdot \nabla \mathrm{T}=\alpha \nabla^{2} T$

In the iron rods:

$\frac{\partial T_{\mathrm{r}}}{\partial t}=\alpha_{\mathrm{r}} \nabla^{2} T_{\mathrm{r}}$

where $\mathbf{U}=\left(U_{\mathrm{R}}, U_{\theta}, U_{\mathrm{Z}}\right)$ is velocity vector, $v$ kinetic viscosity, $t$ time, $\rho$ density, p pressure, $T$ and $T_{\mathrm{r}}$ temperatures of the melt and rods, respectively, $\beta$ thermal expansion coefficient, $g$ the gravity level, $\mathbf{e}_{z}$ a unit vector in the $Z$ direction, and $\alpha, \alpha_{\mathrm{r}}$ the thermal diffusivities of the melt and rods respectively.

The boundary conditions are as follows:

In the melt zone,

at $Z=0$ and $Z=L: \quad \mathbf{U}=0, k \frac{\partial T}{\partial Z}=k_{\mathrm{r}} \frac{\partial T_{\mathrm{r}}}{\partial Z}$

at $R=h(Z): \mathbf{n} \cdot \mathbf{U}=0, \mathbf{n} \cdot \nabla T=0, \mathbf{t}_{\mathrm{z}} \cdot(\boldsymbol{\tau} \cdot \mathbf{n})=-\mathbf{t}_{\mathrm{z}} \cdot \nabla T$,

$\mathbf{t}_{\theta} \cdot(\boldsymbol{\tau} \cdot \mathbf{n})=-\mathbf{t}_{\theta} \cdot \nabla T$

In the iron rods,

at $Z=-L_{\mathrm{r}}: T_{\mathrm{r}}=T_{\mathrm{C}} ;$ at $Z=L+L_{\mathrm{r}}: T_{\mathrm{r}}=T_{\mathrm{H}}$ in the top-heated case (noted as TH)

at $Z=-L_{\mathrm{r}}: T_{\mathrm{r}}=T_{\mathrm{H}} ;$ at $Z=L+L_{\mathrm{r}}: T_{\mathrm{r}}=T_{\mathrm{C}}$ in the bottom-heated case (noted as BH)

at $R=a: \mathbf{n} \cdot \nabla T_{\mathrm{r}}=0$

where $k$ and $k_{\mathrm{r}}$ are the heat conductivities of the melt and rod respectively, $h(Z)$ the radius of the free surface $\mathbf{n}$ the normal vector of the free surface, $\mathbf{t}_{\mathrm{z}}$ and $\mathbf{t}_{\theta}$ are the tangential vectors of the free surface in the $(R, Z)$ plane and $(R, \theta)$ plane respectively, and $\tau$ the stress tensor. In the present study, the driving force of the Marangoni flow is not given a priori. An effective temperature difference between the melt/solid interfaces, $\Delta T_{\mathrm{e}}$, at each time step is calculated a posteriori as

$\Delta T_{\mathrm{e}}=\frac{1}{2 \pi}\left(\int_{0}^{2 \pi} T(a, \theta, 0) \mathrm{d} \theta-\int_{0}^{2 \pi} T(a, \theta, L) \mathrm{d} \theta\right)$

where $T(a, \theta, 0)$ and $T(a, \theta, L)$ is the temperature on the three phase contact lines. The Prandtl number, Reynolds number and Grashof number are respectively defined as

$\operatorname{Pr}=\frac{v}{\alpha}, \quad \mathrm{Bo}=\frac{\rho \mathrm{g} a^{2}}{\sigma}, \quad \operatorname{Re}=\frac{\sigma_{\mathrm{T}}\left|\Delta T_{\mathrm{e}}\right| a}{\rho v^{2}}, \quad \mathrm{Gr}=\frac{\beta g \Delta T_{\mathrm{e}} a^{3}}{v^{2}}$

Due to the experimental fact that dynamic free surface deformations $\left(10^{-6} \mathrm{~m}\right)$ are negligibly small compared to the FHZ size $\left(\mathrm{O}\left(10^{-2} \mathrm{~m}\right)\right)$, dynamic free surface deformations are ignored in the present study. Then the static free surface shape $h(Z)$ is assumed to be non-deformable and axisymmetric to the $Z$-axis which is determined by solving the Young-Laplace equation:

$\frac{1}{h(Z)\left(1+h(Z)^{\prime 2}\right)^{\frac{1}{2}}}-\frac{h(Z)^{\prime \prime}}{\left(1+h(Z)^{\prime 2}\right)^{\frac{3}{2}}}=\frac{a \Delta p}{\sigma}-\frac{\rho g a}{\sigma} Z$

with two boundary conditions and a volume constant:

at $Z=0: h(0)=a ; \quad$ at $Z=L: h(L)=a$

$V=\int_{0}^{L} \pi h(Z)^{2} \mathrm{~d} Z=\pi a^{2} L=2 \pi a^{3}$

In the present study, the non-cylindrical physical domain is transformed into a uniform computational domain through the body-fitted transformation [26]. The governing equations are discretized through the finite volume method on staggered grids in the computational domain. The details of the numerical method can be found elsewhere [18]. The mesh number is $40 \times 40 \times 80$ in $(R, \theta$ and $Z)$ directions, respectively, for the melt zone, and $40 \times 40 \times 40$ for the iron rods. Note that non-uniform staggered grid are adopted in the physical domain to increase the resolution near the boundaries. To validate the code, the present model is simplified to a FHZ model with melt zone only (the mesh number is $40 \times 40 \times 80$ in $(R, \theta$ and $Z)$ directions $)$. The corresponding numerical results are in a good agreement with the benchmark work [22] as shown in Table 2.

\section{Results and discussions}

In the present study, the results for $\mu \mathrm{G}$ case $(\mathrm{Bo}=0, \mathrm{Gr}=0)$, at which the melt zone is cylindrical, was calculated for the reference. The present FHZ $\left(a=3 \mathrm{~mm}, \mathrm{As}=\mathrm{As}_{\mathrm{r}}=2.0, V=2 \pi a^{3} \quad\right.$ and Bo $=1.20$ under $1 \mathrm{G}$ ) has a $S$ shaped free surface with a maximum radius $1.09 a$ and a minimum $0.87 a$ as shown in Fig. 2.

All the cases studied are classified into three groups according to the Grashof number ( $\mathrm{Gr}$ ), the Bond number (Bo) and the heating direction:

Group 1: FHZ with cylindrical free surface:

$(\mathrm{Bo}=0, \mathrm{Gr}=0),(\mathrm{Bo}=0, \mathrm{Gr}>0)$ and $(\mathrm{Bo}=0, \mathrm{Gr}<0)$;

Group 2: FHZ with deformed free surface heated from top:

$(\mathrm{Bo}=1.20, \mathrm{Gr}<0)$ and $(\mathrm{Bo}=1.20, \mathrm{Gr}=0, \mathrm{TH})$;

Group 3: FHZ with deformed free surface heated from bottom, $(\mathrm{Bo}=1.20, \mathrm{Gr}>0)$ and $(\mathrm{Bo}=1.20, \mathrm{Gr}=0, \mathrm{BH})$.

For current $\mathrm{FHZ}$ with non-zero $\mathrm{Gr}$, the flow field is expected to be Marangoni flow dominant due to the small dynamic Bond number $\left(\mathrm{Bo}_{d}=|\mathrm{Gr} / \mathrm{Re}|=0.60<1\right)$.

\subsection{Axisymmetric non-oscillatory flow regime}

As time elapsed, the temperature gradient develops in the melt zone which drives the Marangoni flow, in cooperation with buoyancy for $1 \mathrm{G}$ cases. The flow in the melt zone is axisymmetric and non-oscillatory until $\left|\Delta T_{\mathrm{e}}\right|$ exceeds a certain threshold value. The effects of the free surface shape and the buoyancy on the axisymmetric non-oscillatory temperature and flow fields at $\left|\Delta T_{\mathrm{e}}\right|=0.23 \mathrm{~K}(\mathrm{Re}=351)$, much smaller than the first critical conditions, are shown in Figs. 2 and 3. It should be noted that $Z^{*}$, the horizontal axis of Fig. 3, is the distance from the hot melt/rod interface. In Fig. 2, all isothermals meet the free surface at right angle because of the adiabatic boundary condition at the free surface. In $\mu \mathrm{G}$ case $(\mathrm{Bo}=0, \mathrm{Gr}=0, \mathrm{BH})$ the free surface temperature distributes approximately linearly in $Z^{*}$ direction (Fig. 3a). However, Fig. 2b shows small variation of temperature in the radial direction and on a horizontal cut plane at $Z=0.5 L$, free surface temperature is slightly higher than that at $R=0$ in $\mu \mathrm{G}$ cases. The absolute value of the free surface velocity $\left|U_{z}\right|$ shows a smooth curve with a maximum around $Z^{*}=0.55 L=0.0033 \mathrm{~m}$ as shown in Fig. $3 \mathrm{~b}$.

On the contrary, the distributions of the free surface temperature and velocity in the deformed FHZ under $1 \mathrm{G}$ distinguishably deviate from those of $\mu \mathrm{G}$ case. It can be seen from Fig. 3a that large free surface temperature gradient $\left(-\mathrm{d} T / \mathrm{d} Z^{*}\right)$ appears in the neck zone, $\quad 0.65 L<Z<0.9 L \quad\left(0.0039 \mathrm{~m}<Z^{*}<0.0054 \mathrm{~m}\right)$ for bottomheated case: $\mathrm{Gr}>0$, and $0.0006 \mathrm{~m}<Z^{*}<0.0021 \mathrm{~m}$ for top-heated case: $\mathrm{Gr}<0$. Even in cases of Groups 2 and 3, the base flow is mostly driven by the free surface temperature gradient (Marangoni effect). Then the flow intensity depends on the free surface temperature gradient and the length over which the free surface tension gradient effectively accelerate the flow. The velocity distributions in FHZ (Fig. 2a and c) and on the free surface (Fig. 3b) well 
Table 2

The code validation against Shevtsova [22] for the case of $\operatorname{Pr}=0.02, \operatorname{Re}=2000, \mathrm{Gr}=0$..

\begin{tabular}{|c|c|c|c|c|c|}
\hline & & \multicolumn{2}{|c|}{$\left.\underline{\text { Stream-function }|\psi|}\right|_{\max }$} & \multicolumn{2}{|c|}{$\left|V_{Z}\right|_{\max }$ on free surface } \\
\hline & & As $=1$ & As $=1.2$ & As $=1$ & As $=1.2$ \\
\hline \multirow[t]{2}{*}{ Contact angle $40^{\circ}$} & Shevtsova [22] & 8.42 & 5.61 & 188.61 & 184.46 \\
\hline & Present code & 8.57 & 5.82 & 191.15 & 189.31 \\
\hline \multirow[t]{2}{*}{ Contact angle $90^{\circ}$} & Shevtsova [22] & 15.67 & 12.67 & 201.84 & 205.61 \\
\hline & Present code & 15.74 & 12.74 & 201.97 & 205.84 \\
\hline
\end{tabular}

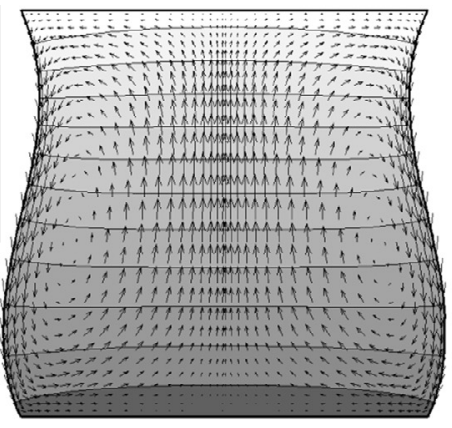

(a)

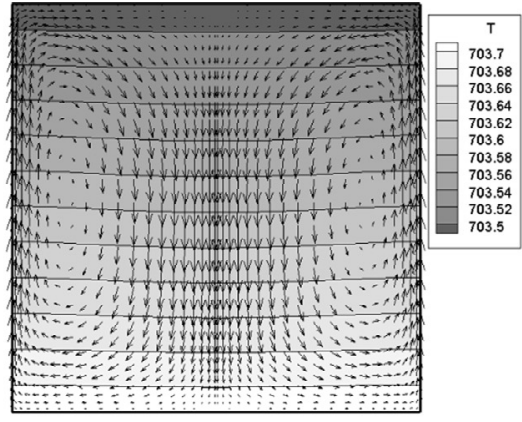

(b)

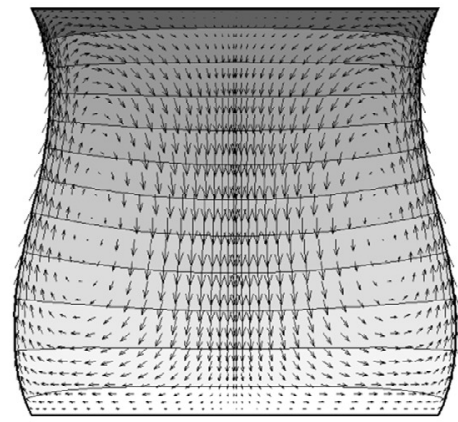

(c)

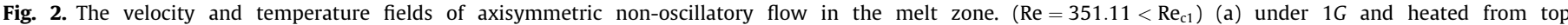
$(\mathrm{Bo}=1.20, \mathrm{Gr}=-210.66)$, (b) under $\mu \mathrm{G}$ and heated from bottom $(\mathrm{Bo}=0, \mathrm{Gr}=0)$ and $(\mathrm{c})$ under $1 \mathrm{G}$ and heated from bottom $(\mathrm{Bo}=1.20, \mathrm{Gr}=210.66)$.

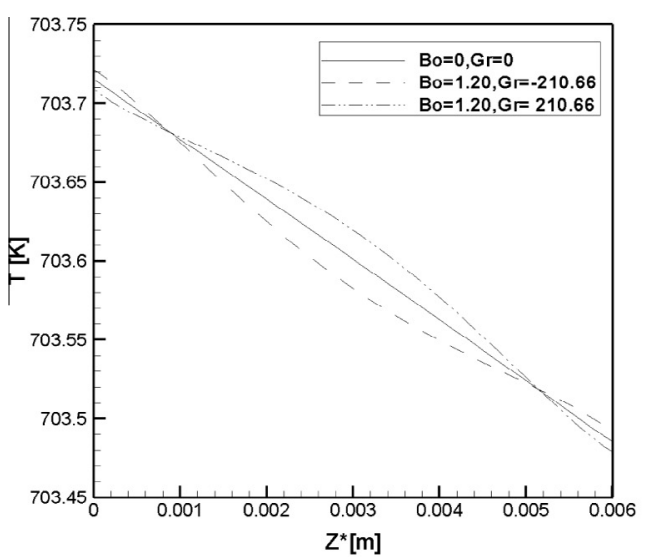

(a)

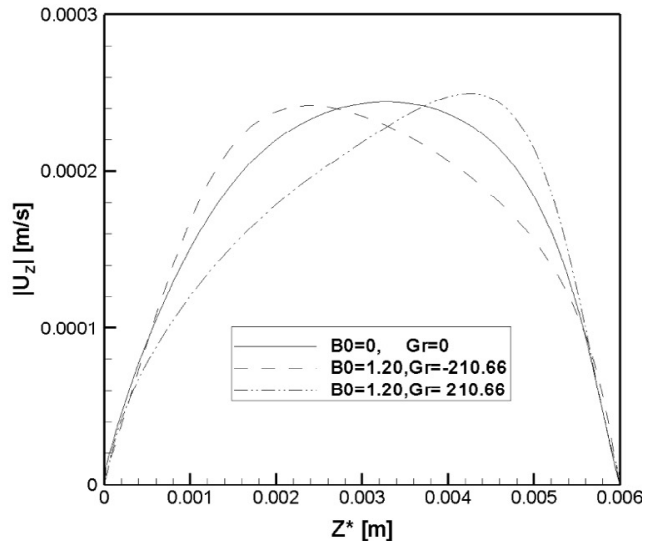

(b)

Fig. 3. (a) The free surface temperatures and (b) the absolute values of surface velocity in the Z-direction as a function of distance from the hot melt/rod interface.

correspond to the free surface temperature distributions (Fig. 3a). Maximum $\left|U_{z}\right|$ in Fig. 3b appears at around $Z=0.62 L\left(Z^{*}=0.38\right.$ $L=0.0023 \mathrm{~m}$ ) for top-heated case and at $Z=Z^{*}=0.70 L=0.0042 \mathrm{~m}$ for bottom-heated case. It should be noted that in all cases, the isothermals near the free surface have positive radial gradient near the mid-height of the melt zone, then the free surface temperature on the mid-height is closer to the temperature of the bottom plate.

\subsection{Time evolution of the absolute value of effective temperature difference $\Delta T_{\mathrm{e}}$}

As mentioned above, a linearly ramped overall temperature difference is applied between both ends of the supporting iron rods. The time evolutions of the absolute value of effective temperature difference $\left(\left|\Delta T_{\mathrm{e}}\right|\right)$ between the upper and lower melt/rod interfaces for all cases studied are shown in Fig. 4. $\left|\Delta T_{\mathrm{e}}\right|$ for all cases are nearly linear in early calculation time span $(t<200 \mathrm{~s})$, but the slope is slightly dependent on the shape of FHZ. In cases of cylindrical
FHZ, the slope $\left(\mathrm{d}\left|\Delta T_{\mathrm{e}}\right| / \mathrm{d} t\right)$ is about $0.21 \mathrm{~K} / \mathrm{min}$. The buoyancy force seems to play a minor role in the cylindrical $\mathrm{FHZ}$ over a wide range of the calculation time span $(t<650 \mathrm{~s})$. In case of deformed $\mathrm{FHZ}$, $\left|\Delta T_{\mathrm{e}}\right|$ is always about $3 \%$ larger than those of the cylindrical FHZ. The difference can be attributed to the non-uniform cross sectional area of the melt zone. During the entire calculation time span, the slope changes at least twice, accompanied by the flow transitions, but the changes cannot significantly be clarified in Fig. 4. In order to detect flow transitions during the entire calculation time span, local velocities and temperatures are plotted as a function of $\left|\Delta T_{\mathrm{e}}\right|$, instead of time, as shown in Figs. 5 and 6. It can be seen that the buoyant-Marangoni flow in FHZ of molten tin is axisymmetric stationary until $\left|\Delta T_{e}\right|$ exceeds a certain threshold. At a first threshold value, the flow changes from an axisymmetric non-oscillatory flow to a 3D asymmetric non-oscillatory flow. At further larger $\left|\Delta T_{\mathrm{e}}\right|$ (a second threshold value), 3D oscillatory flow sets on. In the following sections, each regime of the flow transitions will be described in detail. 


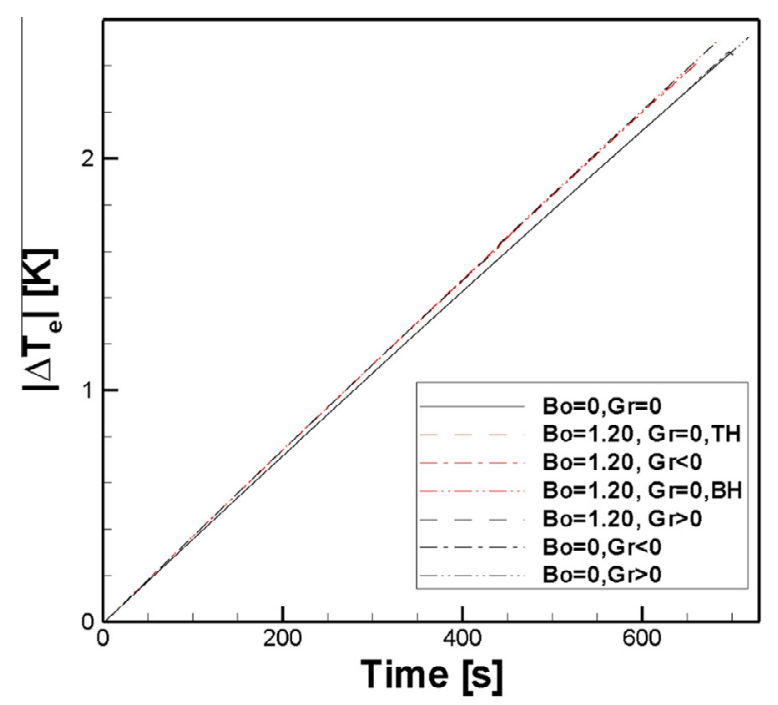

Fig. 4. Time evolution of the absolute value of the effective temperature difference between the melt/rod interfaces.

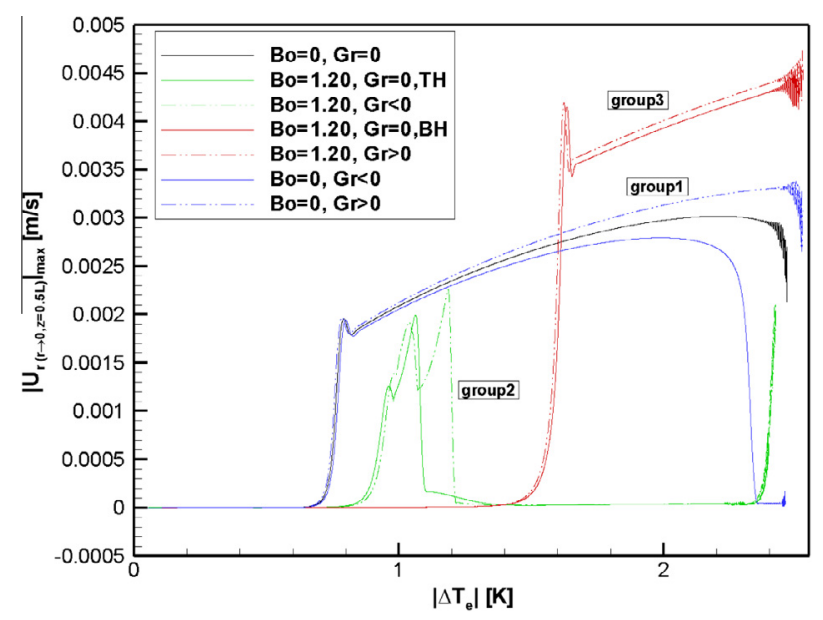

Fig. 5. Evolutions of the maximum absolute value of radial velocity close to the axis at mid-plane of the melt zone against $\left|\Delta T_{\mathrm{e}}\right|$ between the melt/rod interfaces.

\subsection{Asymmetric non-oscillatory flow regime}

After the overall ramping temperature difference started, axisymmetric non-oscillatory flow increases its intensity as shown in Fig. 6 in $\mu \mathrm{G}$ case. At the first critical temperature difference $\left|\Delta T_{\mathrm{e}}\right|_{\mathrm{c} 1}$, or the first critical Reynolds number $\mathrm{Re}_{\mathrm{c} 1}$, the flow becomes unstable and a flow exhibits a transition to a 3D asymmetric nonoscillatory flow. As shown in the following figures and Table 3, $\left|\Delta T_{\mathrm{e}}\right|_{\mathrm{c} 1}$ or $\operatorname{Re}_{\mathrm{c} 1}$ strongly depends on the free surface shape and the heating direction. Due to the transient nature of the present study, detection of the critical point is more difficult than our previous works $[18-20,26]$. In those studies, transient calculations were conducted for different but constant Re to obtain the growth rate constants of 3D disturbances as a function of Reynolds number. Then the critical $R_{c 1}$ is determined as the value of Re at which the growth rate constant becomes zero. In the present study, the first bifurcation point is determined through the local free surface temperature which is identical to that through the local free surface azimuthal velocity [21].

\subsubsection{Group $1(B o=0)$}

As mentioned above, it is rather difficult to detect the flow transition points from the ever changing local temperatures and velocities in the present work. However, at $\operatorname{Re}=770\left(\left|\Delta T_{\mathrm{e}}\right|=0.50 \mathrm{~K}\right)$, local free surface temperatures of the cylindrical $\mathrm{FHZ}$ under $\mu \mathrm{G}$ ( $\mathrm{Bo}=0, \mathrm{Gr}=0)$ show distinguishable deviations $\left(1.0 \times 10^{-4} \mathrm{~K}\right)$ from the axisymmetric distribution. The cold plume flows down slightly apart from the $Z$-axis and cools one side of the free surface to create a minimum surface temperature on one side of FHZ and a higher temperature area on the opposite side. This disturbance grows with time and a temperature and velocity distribution appear pattern with an azimuthal wave number of $m=1$ (see Fig. 7). Temperature and vectors on the horizontal cut-plane at $Z=0.5 \mathrm{~L}$ (mid-plane) is characterized by its azimuthal wave number $m=1$, since it has single line of symmetry (or symmetric plane) $P_{S Y}$, non-zero $\left|U_{R}\right|$ near the axis, and splitting of the local free surface temperatures and velocities (see Figs. 5 and 6). At larger $\left|\Delta T_{\mathrm{e}}\right|$ the free surface temperature at $\theta=0$ becomes lower than those at $\theta=\pi / 2$ and $\theta=3 \pi / 2$. This corresponds to splitting of the higher temperature zone into two separate zones like Fig. 9a which still holds the characteristics of $m=1$ mode.

For the cylindrical FHZ ( $\mathrm{Bo}=0, \mathrm{Gr}<0, \mathrm{TH})$, the evolution of the 3D asymmetric non-oscillatory flow is qualitatively the same as the $\mu \mathrm{G}$ case except for the different magnitudes of the temperatures and velocities (see Figs. 5 and 8). Moreover, for both cases, the well developed 3D asymmetric non-oscillatory flow with $m=1$ dominates until the second flow transition to an oscillatory flow sets on.

On the other hand, for the cylindrical $\mathrm{FHZ}(\mathrm{Bo}=0, \mathrm{Gr}>0, \mathrm{BH})$, initially the 3D asymmetric stationary flow with $m=1$ (Fig. 7) sets on and gradually deforms its pattern to that of Fig. 9a. The temperature and flow fields of Fig. 9a holds the character of $m=1$ since it has only one line of symmetry. However it also can be recognized as an incomplete (or deformed) $m=2$ since it has two local maxima of free surface temperature. With a further increase of $\left|\Delta T_{\mathrm{e}}\right|$

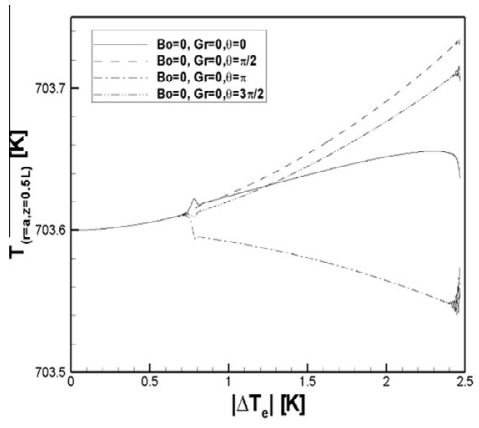

(a)

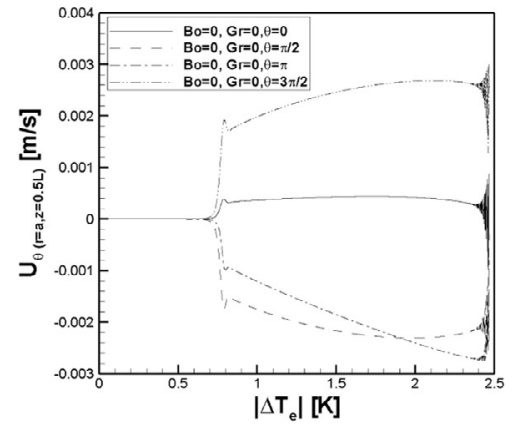

(b)

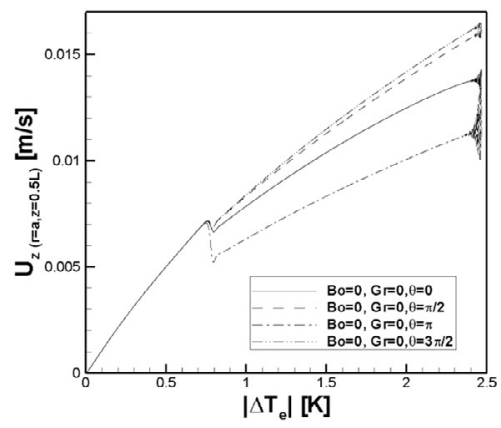

(c)

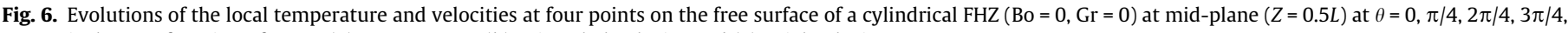
respectively, as a function of $\left|\Delta T_{\mathrm{e}}\right|$ : (a) temperature, (b) azimuthal velocity and (c) axial velocity. 
Table 3

The critical conditions of bifurcation to 3D asymmetric stationary flow.

\begin{tabular}{|c|c|c|c|c|c|c|c|c|}
\hline & & \multicolumn{3}{|c|}{ Present work } & \multicolumn{4}{|c|}{ Lappa et al. [26] } \\
\hline & & \multicolumn{3}{|c|}{$\begin{array}{l}\text { Molten tin, Fluid A and Fluid B Pr }=0.009(B o=1.20 \\
\text { under } 1 G)\end{array}$} & \multicolumn{2}{|c|}{$\begin{array}{l}\text { Si melt: } \operatorname{Pr}=0.01(\mathrm{Bo}=0.85 \\
\text { under } 1 \mathrm{G})\end{array}$} & \multicolumn{2}{|c|}{$\begin{array}{l}\text { Ga melt: } \mathrm{Pr}=0.021(\mathrm{Bo}=2.08 \\
\text { under } 1 \mathrm{G})\end{array}$} \\
\hline & & $\operatorname{Re}_{\mathrm{c} 1}$ & $m_{\mathrm{c} 1}$ & Mode change & $\operatorname{Re}_{\mathrm{c} 1}$ & $m_{\mathrm{c} 1}$ & $\operatorname{Re}_{\mathrm{c} 1}$ & $m_{\mathrm{c} 1}$ \\
\hline \multirow[t]{3}{*}{ Group 1} & $\mathrm{Bo}=0, \mathrm{Gr}=0$ & 770 & 1 & & 762 & 1 & 816 & 1 \\
\hline & $\mathrm{Bo}=0, \mathrm{Gr} \neq 0, \mathrm{TH}$ & 770 & 1 & $m=2$ at $\operatorname{Re} \geqslant 3053$ & 768 & 1 & 874 & 1 \\
\hline & $\mathrm{Bo}=0, \mathrm{Gr} \neq 0, \mathrm{BH}$ & 770 & 1 & & 746 & 1 & 770 & 1 \\
\hline \multirow[t]{2}{*}{ Group $2(\mathrm{TH})$} & Bo $\neq 0, \mathrm{Gr}<0$ & 976 & 2 & & 831 & 2 & 2162 & 2 \\
\hline & $\mathrm{Bo} \neq 0, \mathrm{Gr}=0$ & 976 & 2 & & & & & \\
\hline \multirow[t]{2}{*}{ Group $3(\mathrm{BH})$} & $\mathrm{Bo} \neq 0, \mathrm{Gr}>0$ & 1220 & 1 & & 939 & 1 & $-{ }^{*}$ & \\
\hline & $\mathrm{Bo} \neq 0, \mathrm{Gr}=0$ & 1220 & 1 & & & & & \\
\hline
\end{tabular}

* A direct transition to a 3D oscillatory flow is reported for this case.
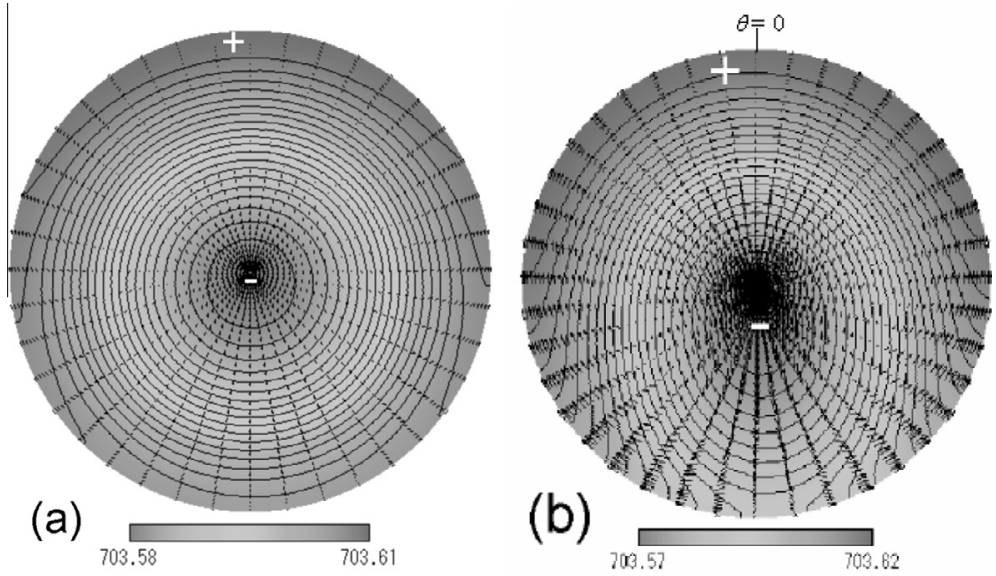

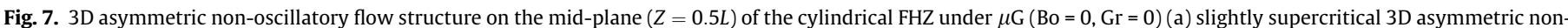
oscillatory flow with $m=1$ and (b)3D non-oscillatory flow with $m=1$ at $\left|\Delta T_{\mathrm{e}}\right|=1.0 \mathrm{~K}$. (+: higher temperature, - : lower temperature).

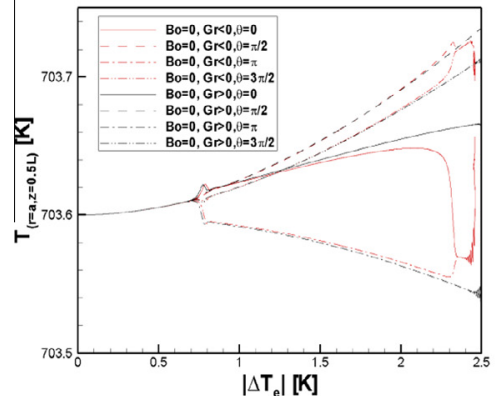

(a)

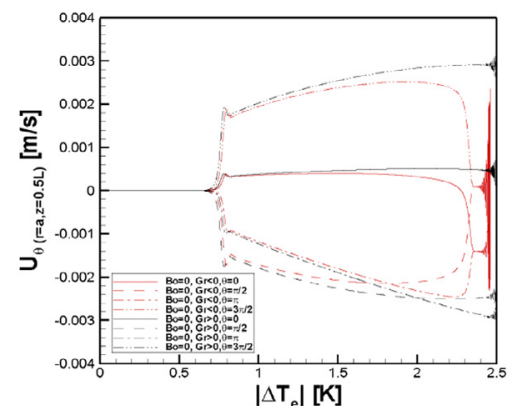

(b)

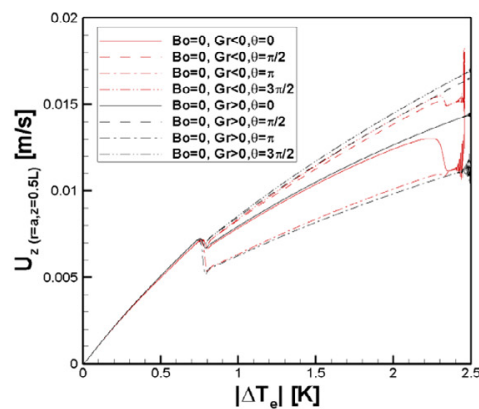

(c)

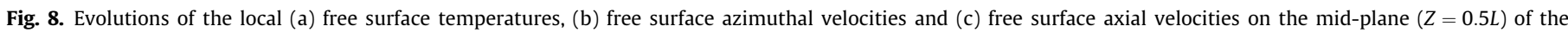
cylindrical $\mathrm{FHZ}(\mathrm{Bo}=0, \mathrm{Gr} \neq 0)$ at $\theta=0, \pi / 2, \pi, 3 \pi / 2$ respectively as a function of $\left|\Delta T_{\mathrm{e}}\right|$.

the flow pattern of $m=1$ (Fig. 9a) becomes unstable and transforms to another non-oscillatory flow mode of $m=2$ (Fig. 9b) at about $\left|\Delta T_{\mathrm{e}}\right|=2.00 \mathrm{~K}(\mathrm{Re}=3020)$. It should be noted that the free surface flow does not follow the Marangoni effect, i.e., velocity vectors near the surface indicate that surface moves from cold spots to hot spots against the Marangoni effect. This kind of transition between 3D non-oscillatory flows was also found in short FHZ (As $=0.8$ ) of molten tin under $\mu \mathrm{G}$ [27].

\subsubsection{Group 2 (top-heated deformed $F H Z$ )}

As shown in Fig. 10a, local free surface temperatures on the mid-plane of a deformed $\mathrm{FHZ}(\mathrm{Bo}=1.20, \mathrm{Gr}=0, \mathrm{TH})$ continue decreasing from their initial values with increasing $\left|\Delta T_{\mathrm{e}}\right|$. Since the free surface temperature on the mid-plane is closer to that of the lower melt/rod interface (see Fig. 3a), the temperature distribution on the mid-plane (see Fig. 11a) is different from those of other cases, i.e. highest temperature appears in a subsurface region and the free surface temperature is slightly lower. At $\operatorname{Re}_{\mathrm{c} 1}=976$ $\left(\left|\Delta T_{e}\right|=0.64 \mathrm{~K}\right)$, the $3 \mathrm{D}$ axisymmetric non-oscillatory flow is broken by a developing 3D weak disturbance which looks like an asymmetric mode of $m=1$ (see Fig. 11b). The first transition is accompanied by a quick increase of the maximum $\left|U_{R}\right|$ near the Z-axis at mid-plane of the FHZ (see Fig. 5) and splitting of local free surface temperatures and velocities (see Fig. 10). Further evolution of the 3D asymmetric non-oscillatory flow proceeds as shown in Fig. 11b-e. After taking a transition state which looks like an 

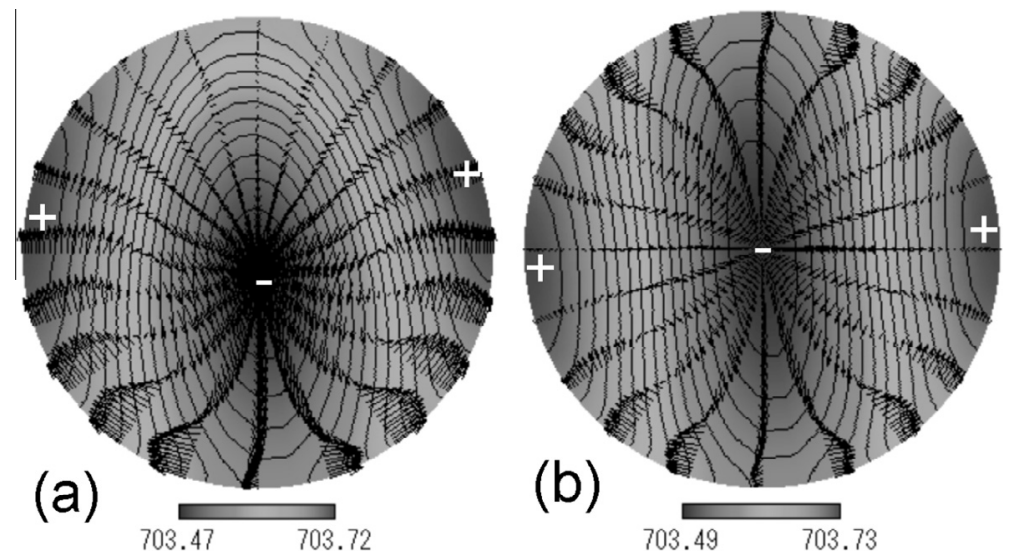

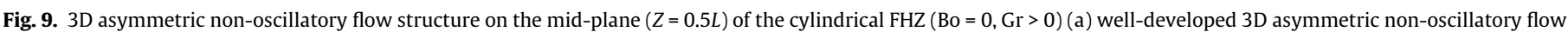
with $m=1$, (b) 3D asymmetric non-oscillatory flow right after the transition from mode $m=1$ to mode $m=2$. (+: higher temperature, - : lower temperature).

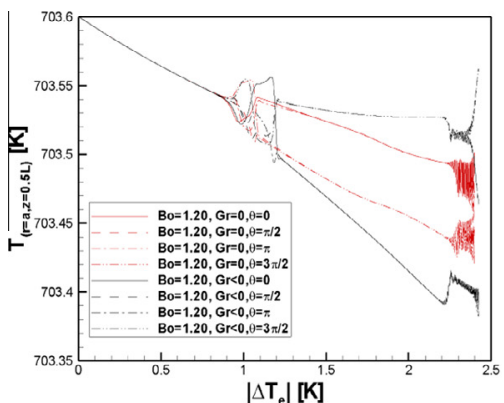

(a)

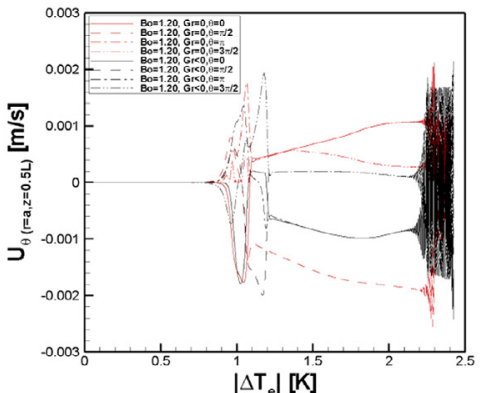

(b)

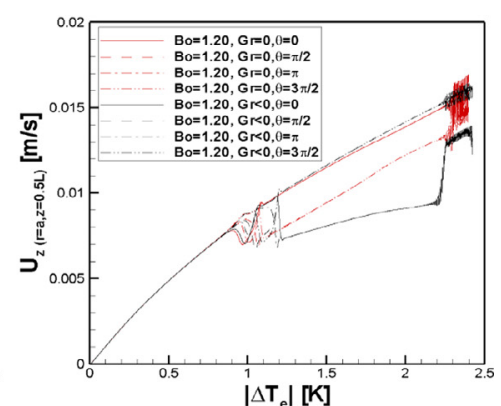

(c)

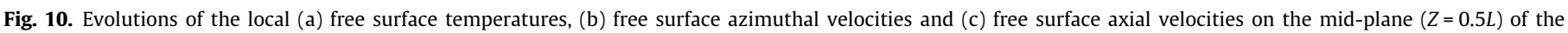
deformed $\mathrm{FHZ}(\mathrm{Bo}=1.20, \mathrm{Gr} \leqslant 0, \mathrm{TH})$ at $\theta=0, \pi / 2, \pi, 3 \pi / 2$ respectively as a function of $\left|\Delta T_{e}\right|$.

asymmetric mode of $m=2$ (see Fig. 11c), another 3D disturbance with $m=1$ appears instantaneously (see Fig. 11d). With a further $\left|\Delta T_{e}\right|$ increase, the hotter crescent zone of $m=1$ splits (see Fig. 11e) and finally transforms to a 3D asymmetric non-oscillatory flow of $m=2$ (see Fig. 11f). The peaks of the local maximum $\left|U_{R}\right|$ near the Z-axis on the mid-plane (Fig. 5) also correspond to the process. From our viewpoint, the critical mode in this case is assigned to be $m=2$ despite the initial weak ( $m=1)$-like 3D asymmetric disturbance. For the deformed FHZ $(\mathrm{Bo}=1.20, \mathrm{Gr}<0)$ in group 2, evolution of the 3D asymmetric non-oscillatory flow proceeds in a similar way as Fig. 11. However, when the second $(m=1)$-like pattern (similar to Fig. 11d) appears, it rotates counterclockwise about $\pi / 2$ with respect to the $Z$-axis before proceeding to the rest of the transition scenario from (e) to (f) of Fig. 11 $(\mathrm{Bo}=1.20, \mathrm{Gr}=0, \mathrm{TH})$, which causes the second large peak of $\left|U_{R}\right|_{R \rightarrow 0}$ in Fig. 5 and of $T, U_{R}$ and $U_{Z}$ on the free surface shown by the black lines in Fig. 10.

\subsubsection{Group 3 (bottom-heated deformed FHZ)}

For both cases of deformed FHZs $(\mathrm{Bo}=1.20, \mathrm{Gr}=0, \mathrm{BH})$ and $(\mathrm{Bo}=1.20, \mathrm{Gr}>0, \mathrm{BH})$, transitions to $3 \mathrm{D}$ asymmetric non-oscillatory flow are qualitatively the same, see Figs. 5 and 12 . At $\operatorname{Re}_{\mathrm{c} 1}=1220\left(\left|\Delta T_{e}\right|=0.79 \mathrm{~K}\right)$, the 3D axisymmetric non-oscillatory flow is unstable to a developing 3D weak disturbance of $m=1$, and then 3D asymmetric non-oscillatory flow as shown in Fig. 13 is quickly developed and is stably maintained until the 3D oscillatory flow sets on.

\subsubsection{Comparison with previous results}

The critical conditions for the onset of 3D asymmetric nonoscillatory flow for all cases studied in the present study $\left(a=3 \mathrm{~mm}, \mathrm{As}=\mathrm{As}_{\mathrm{r}}=2.0, \mathrm{As}=\mathrm{As}_{\mathrm{r}}=2.0, \mathrm{Pr}=0.009, \mathrm{Bo}=1.20\right)$ are tabulated in Table 3, comparing with the results of Lappa et al. [26] for FHZ of Silicon $(a=5 \mathrm{~mm}, \mathrm{As}=2.0, \mathrm{Pr}=0.01, \mathrm{Bo}=0.85$ ) and of Gallium ( $a=5 \mathrm{~mm}, \mathrm{As}=2.0, \operatorname{Pr}=0.04$, Bo=2.08). It can be seen that $\mathrm{Re}_{\mathrm{c} 1}$ for cylindrical $\mathrm{FHZ}$ is close to those for cylindrical FHZ of Si but significantly smaller than those for cylindrical FHZ of Gallium. The deviations can be justified by linear stability analyses of Wanschura et al. [4] and Levenstam et al. [6], i.e. $\operatorname{Re}_{\mathrm{c} 1}$ for FHZ of low-Pr fluids with $\mu \mathrm{G}$ slightly increases with increasing Pr. When free surface deformation and buoyancy are taken into consideration, i.e. deformed $\mathrm{FHZ}$ in groups $2-3, \mathrm{Re}_{\mathrm{c} 1}$ is much larger than those for cylindrical FHZ in group 1 for both heating directions. This suggests the significant stabilization effect of the free surface deformation.

The base flow is further stabilized by heating from bottom. The critical azimuthal wave number is $m_{\mathrm{c} 1}=1$ for groups 1 and 3 . However, for group 2 (top-heated deformed FHZ) $m_{\mathrm{c} 1}=2$ regardless the presence of buoyancy. These trends are in accordance with the results of Lappa et al. [26]. However, the present results show a unique transition between 3D asymmetric non-oscillatory flows at much higher Re in a cylindrical FHZ heated from top with buoyancy $(\mathrm{Bo}=0, \mathrm{Gr}<0, \mathrm{TH})$. The critical conditions summarized in Table 3 suggest that the larger gravity-induced free surface deformation of FHZ causes the more stabilization against three dimensional disturbances.

Linear stability analysis of Kuhlmann et al. predicts the influence of Bo and $\mathrm{Gr}$ on $\operatorname{Re}_{\mathrm{c} 1}$ for $\mathrm{FHZ}$ of $(\operatorname{Pr}=0.023$, $A s=1.0$, $\mathrm{Bo}_{d} / \mathrm{Bo}=0.27$ ) in Fig. 3b of [24]. In their study, when the FHZ is heated from top, $\operatorname{Re}_{\mathrm{c} 1}$ shows a local minimum at small Bo and further increase of Bo, and when the FHZ is heated from bottom, $\mathrm{Re}_{\mathrm{c} 1}$ monotonically increases with increasing Bo. The plot provides 

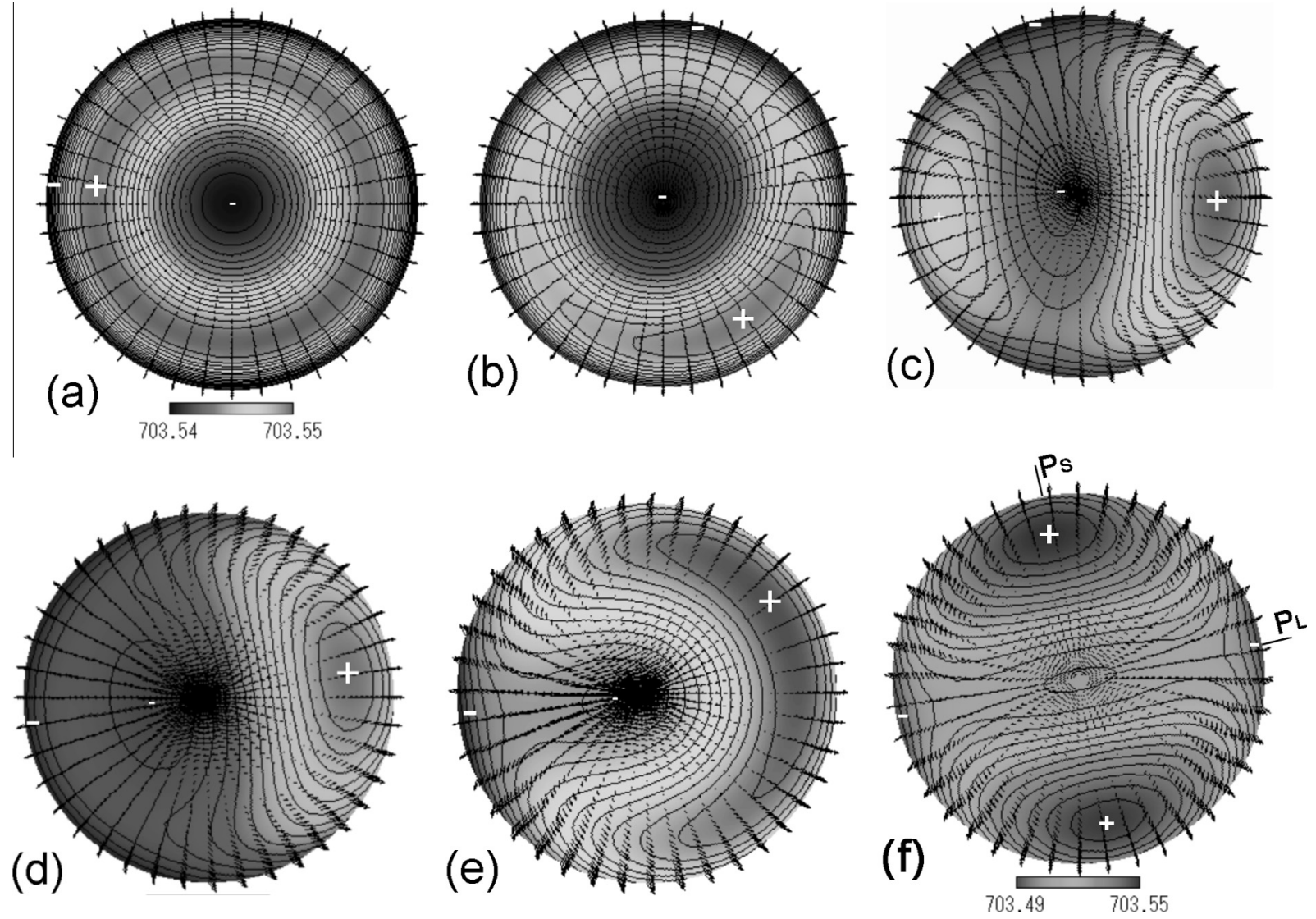

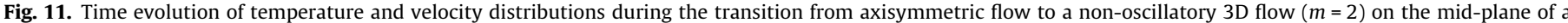
deformed FHZ heated from top ( $\mathrm{Bo}=1.20, \mathrm{Gr}=0$ ).

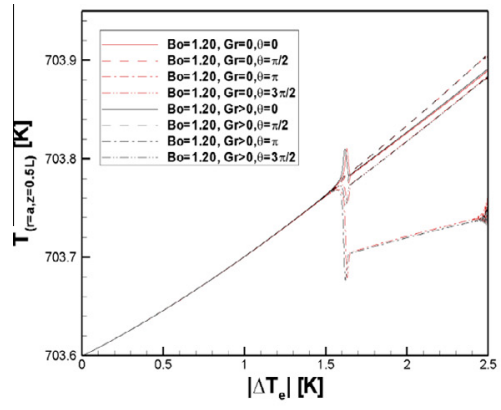

(a)

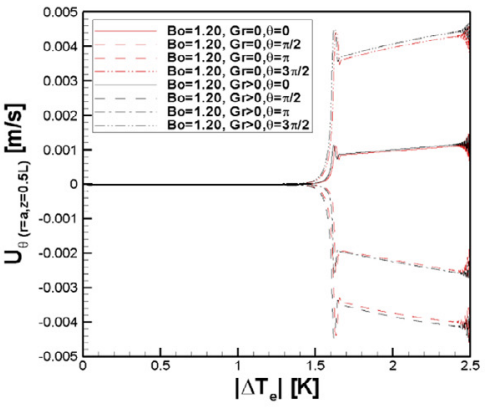

(b)

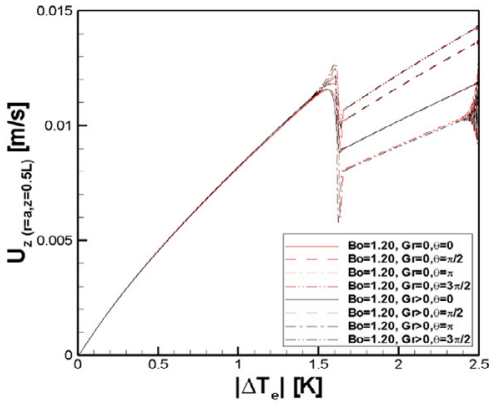

(c)

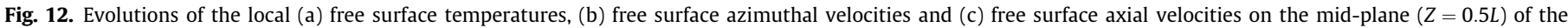
deformed $\mathrm{FHZ}(\mathrm{Bo}=1.20, \mathrm{Gr}>0, \mathrm{BH})$ at $\theta=0, \pi / 2, \pi, 3 \pi / 2$ respectively as a function of $\left|\Delta T_{\mathrm{e}}\right|$.

$\operatorname{Re}_{\mathrm{c} 1}(\mathrm{Bo}=1.20, \mathrm{Gr}=0) / \operatorname{Re}_{\mathrm{c} 1}(\mathrm{Bo}=0, \mathrm{Gr}=0)=0.96$ for the case heated from top and 1.33 for the case heated from top, and $\operatorname{Re}_{\mathrm{c} 1}(\mathrm{Bo}=1.20, \quad \mathrm{Gr} \neq 0) / \operatorname{Re}_{\mathrm{c} 1}(\mathrm{Bo}=1.20, \quad \mathrm{Gr}=0)=1.02$ for the heated from top and 0.97 for the case heated from bottom. The present study on different conditions $(\mathrm{Pr}=0.009$, $\mathrm{As}=2.0$ and $\mathrm{Bo}_{d} / \mathrm{Bo}=0.50$ ) show the trend of $\operatorname{Re}_{\mathrm{c} 1}$ as a function of Bo.

\section{4. $3 D$ oscillatory flow regime}

At a certain threshold of temperature difference, $\left|\Delta T_{\mathrm{e}}\right|_{\mathrm{c} 2}$ or $\mathrm{Re}_{\mathrm{c} 2}$, the 3D asymmetric non-oscillatory flow in low-Pr FHZ becomes unstable and starts oscillations around the $Z$-axis. At $\operatorname{Re}_{\mathrm{c} 2}$, the oscillation amplitudes of local temperatures are very small but increase in $\left|\Delta T_{\mathrm{e}}\right|$ enlarge the amplitudes.
In the present work, the maximum absolute value of radial velocities at the axis on the mid-plane of the $\mathrm{FHZ}$ is chosen as the most sensitive indicator of the onset of oscillatory instability instead the local temperatures on the free surface. The reason is that the temperature oscillations start much later than the radial velocity's oscillation on the $Z$-axis in the present cases. The critical temperature difference was defined as the effective temperature difference $\left|\Delta T_{\mathrm{e}}\right|$ at which the amplitude of the indicator velocity's oscillation amplitude exceeds $1.0 \times 10^{-5} \mathrm{~m} / \mathrm{s}$. The azimuthal wave number of the 3D oscillatory flow $\left(m_{\mathrm{c} 2}\right)$ is the same as that of the well-developed 3D non-oscillatory flow prior to the start of oscillations. The critical conditions for the second bifurcation in the present FHZ are tabulated in Table 4 . In Table $4, m_{\mathrm{c} 2}$ is the azimuthal wave number at the second critical point $\left(\operatorname{Re}_{\mathrm{c} 2}\right)$, the oscillation 

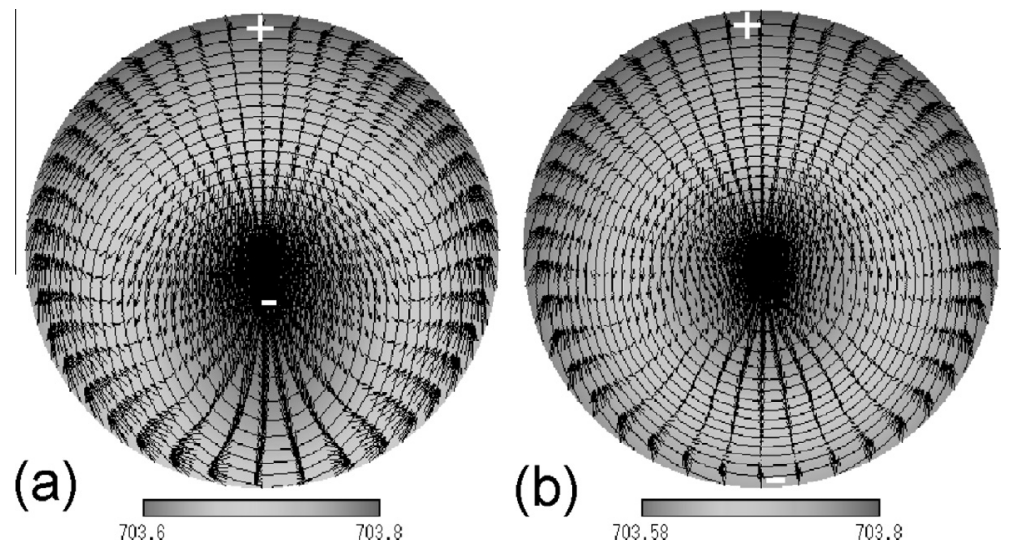

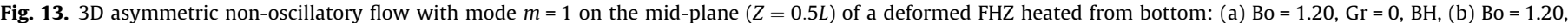
$\mathrm{Gr}>0$, BH. (+: higher temperature, -: lower temperature).

Table 4

The critical conditions for the second bifurcation.

\begin{tabular}{llllll}
\hline & & $\mathrm{Re}_{\mathrm{c} 2}$ & $m_{\mathrm{c} 2}$ & Mode & $f_{\mathrm{c}}(\mathrm{Hz})$ \\
\hline Group 1 & $\mathrm{Bo}=0, \mathrm{Gr}=0$ & 3278 & 1 & $1-\mathrm{T}$ & 0.56 \\
& $\mathrm{Bo}=0, \mathrm{Gr}<0, \mathrm{TH}$ & 3611 & 2 & $2-\mathrm{T}$ & 0.56 \\
& $\mathrm{Bo}=0, \mathrm{Gr}>0, \mathrm{BH}$ & 3467 & 1 & $1-\mathrm{T}$ & 0.48 \\
Group 2 & $\mathrm{Bo}=1.20, \mathrm{Gr}<0, \mathrm{TH}$ & 3167 & 2 & $2-\mathrm{T}$ & 0.45 \\
& $\mathrm{Bo}=1.20, \mathrm{Gr}=0, \mathrm{TH}$ & 3122 & 2 & $2-\mathrm{T}$ & 0.48 \\
Group 3 & $\mathrm{Bo}=1.20, \mathrm{Gr}>0, \mathrm{BH}$ & 3444 & 1 & $1-\mathrm{T}$ & 0.50 \\
& $\mathrm{Bo}=1.20, \mathrm{Gr}=0, \mathrm{BH}$ & 3400 & 1 & $1-\mathrm{T}$ & 0.48 \\
\hline
\end{tabular}

mode " $m$ - $T$ " means the flow and temperature fields on the midplane is characterized by an azimuthal wave number " $m$ ", and one of the lines of symmetry is twisting back and forth in azimuthal direction around the $Z$-axis."

Li et al. [28] studied the three dimensional structures of the oscillating disturbances of " $\mathrm{m}$ - $\mathrm{T}$ " and other oscillation modes, such as " $m$-p" (or " $m+1$ ") (the center of the pattern of $m$ moves back and forth on a linear line like a pendulum). The oscillation mode first reported by Levenstam and Amberg [5] for floating half zone of $(\operatorname{Pr}=0.01$, As $=1.0)$ corresponds to this " $2-p$ " (or " $2+1$ ") mode. Li et al. [21] also reported combined oscillation modes at much higher super-criticalities, such as "2-T+R" (whole temperature and velocity fields with 2-T type oscillation rotates around the Zaxis) and " $1-\mathrm{T}+1$ " (center of the temperature and velocity fields showing 2-T type oscillation moves like a pendulum). All
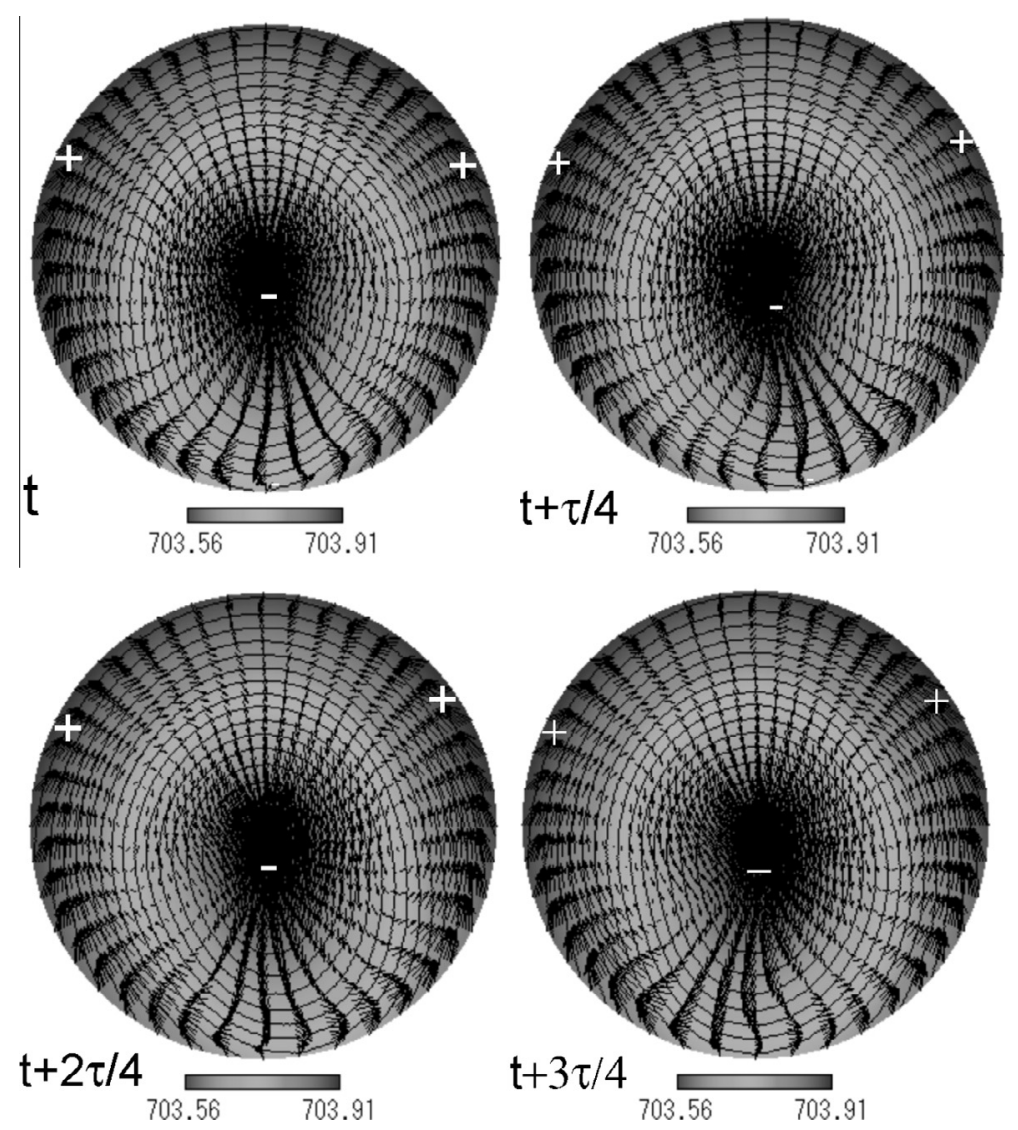

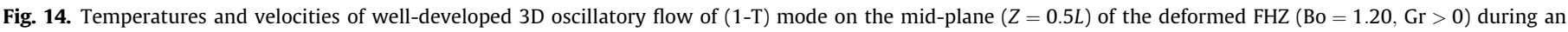
oscillatory period $(\tau)$ in a time interval of $\tau / 4$ (+: higher temperature, - : lower temperature). 

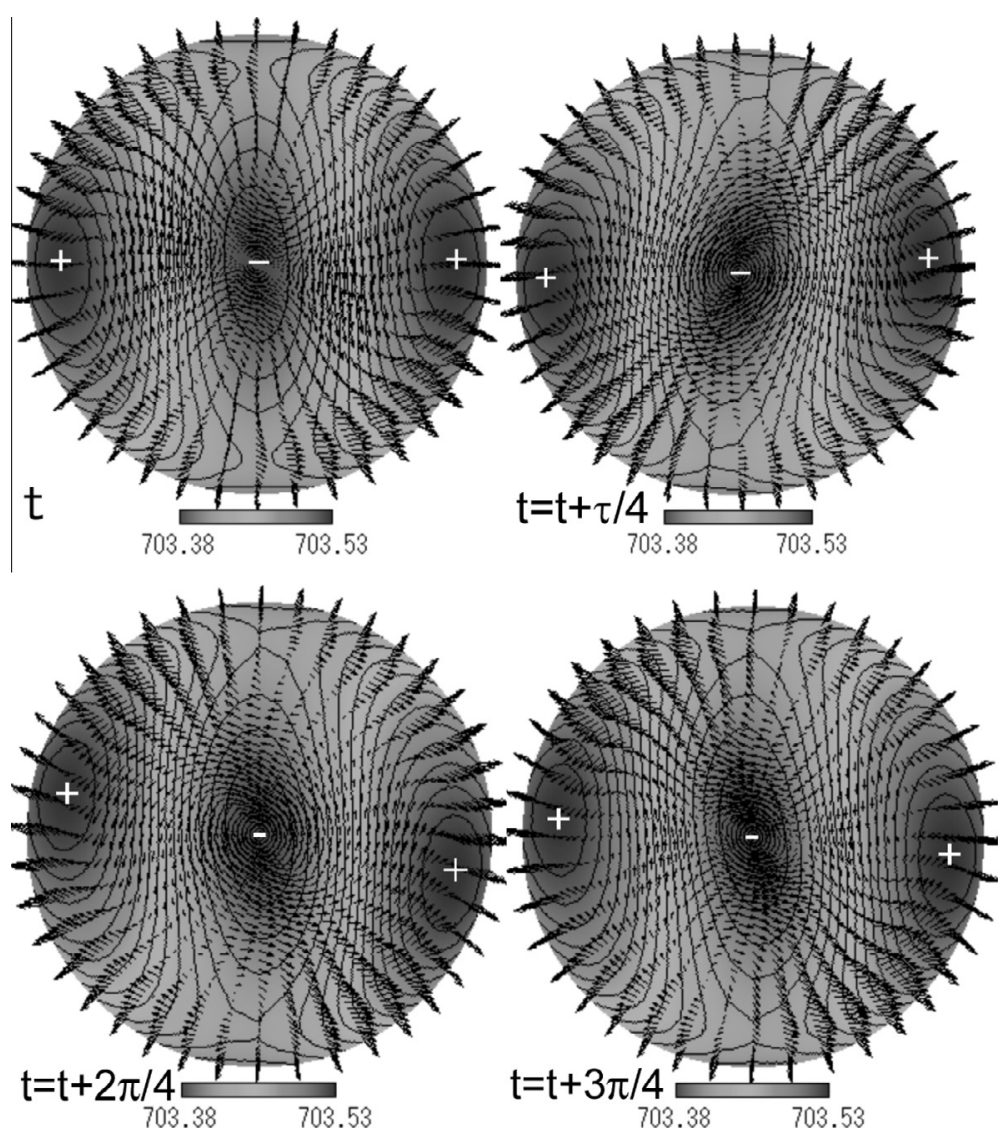

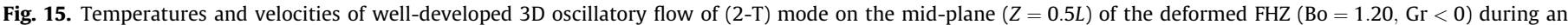
oscillatory period $(\tau)$ in a time interval of $\tau / 4$ (+: higher temperature, - : lower temperature).

oscillations met in the present simulations take twisting mode, either 1 -T or $2-\mathrm{T}$, at the second critical point and slightly supercritical states.

Fig. 14 shows temperature and velocity distributions on the mid-plane of a (1-T) mode oscillation at four instances during an oscillation period in a deformed $\mathrm{FHZ}$ heated from bottom $(\mathrm{Bo}=1.20, \mathrm{Gr}>0, \mathrm{BH})$. The temperature and flow fields at each instance keep the characteristic pattern of $m=1$ which is similar to the 3D well-developed asymmetric non-oscillatory flow of $m_{\mathrm{c} 1}=1$, although it is slightly changing with time. At four different instants during an oscillation period, the torsional disturbance induces an oscillatory motion in the temperature and flow fields in the azimuthal direction with respect to the symmetry plane of $\mathrm{P}_{\mathrm{SY}}$ (see Fig. $7 \mathrm{~b}$ ). The flow around the axis periodically alternates its azimuthal direction between anticlockwise and clockwise directions.

Fig. 15 shows characteristics of a (2-T) mode oscillation by four snapshots of temperature and velocity vectors on the mid-plane of a deformed $\mathrm{FHZ}$ heated from top $(\mathrm{Bo}=1.20, \mathrm{Gr}<0, \mathrm{BH})$ during one period of oscillation. The temperature and flow field at each instance still holds the characteristic pattern of $m=2$, although it is largely deforming with time.

As shown in Table 4, 3D oscillatory flow in cylindrical FHZ under $\mu \mathrm{G}(\mathrm{Bo}=0, \mathrm{Gr}=0)$ is the torsional oscillation mode of (1-T). The oscillation mode is (1-T) for all bottom-heated FHZ and (2-T) for top-heated $\mathrm{FHZ}$, regardless the value of Bo and Gr. It should be noted that the free surface flow is not governed by the Marangoni effect, i.e. velocity vectors near the surface indicate that the free surface flow moves from cold spots to hot spots against the Marangoni effect at almost all instance of oscillations.
The results in Table 4 indicate that the $\mathrm{Re}_{\mathrm{c} 2}$ for cylindrical FHZ with non-zero $\mathrm{Gr}$ is larger than $\mathrm{Re}_{\mathrm{c} 2}$ for cylindrical $\mathrm{FHZ}$ under $\mu \mathrm{G}$ ( $\mathrm{Bo}=0, \mathrm{Gr}=0)$, regardless the heating direction. On the other hand, $\mathrm{Re}_{\mathrm{c} 2}$ for the deformed FHZ heated from top ( $\left.\mathrm{Bo}=1.20, \mathrm{Gr} \leqslant 0\right)$ is smaller than that for the cylindrical $\mathrm{FHZ}$ under $\mu \mathrm{G} \quad(\mathrm{Bo}=0$, $\mathrm{Gr}=0$ ), while $\mathrm{Re}_{\mathrm{c} 1}$ for the deformed $\mathrm{FHZ}$ heated from bottom ( $\mathrm{Bo}=1.20, \mathrm{Gr} \geqslant 0$ ) is larger than that for $\mu \mathrm{G}$.

The present results indicate that the combined effects of free surface deformation and buoyancy destabilize the three-dimensional non-oscillatory flow if the FHZ is heated from top, and stabilize if the deformed FHZ is heated from bottom. Moreover, in the same group, the presence of buoyancy always causes further stabilization. However, for all the cases studied in the present work, the effects of free surface deformation and buoyancy on $\mathrm{Re}_{\mathrm{c} 2}$ are much less than those on $\operatorname{Re}_{\mathrm{c} 1}$.

\section{Conclusions}

Gravity affects the stability of Marangoni flow in floating half zone of low-Pr fluids through two different factors, i.e., the buoyancy and the static deformation of the free surface shape. In the present study, influence of these two factors are evaluated by unsteady three-dimensional (3D) simulations based on a realistic model of floating half zone of molten tin $(a=3 \mathrm{~mm}$, As $=A s_{r}=2.0, V=2 \pi \mathrm{a}^{3}$ and $\mathrm{Bo}=1.20$ under $1 \mathrm{G}$ ) under a ramped temperature difference $(1.19 \mathrm{~K} / \mathrm{min})$ between the top and bottom ends of two iron supporting rods. All the cases studied in the present study are classified into three groups according to the Grashof number ( $\mathrm{Gr}$ ), the Bond number (Bo) and the heating direction: the cylindrical $\mathrm{HZ}$ with $\mu \mathrm{G}$ case $(\mathrm{Bo}=0, \mathrm{Gr}=0)$ and two accompanied 
cylindrical HZs with buoyancy only $(\mathrm{Bo}=0, \mathrm{Gr} \neq 0$ ), the deformed FHZ under $1 G$ in bottom-heated situation $(\mathrm{Bo}=1.20, \mathrm{Gr} \geqslant 0, \mathrm{BH})$ and the deformed $\mathrm{FHZ}$ under $1 \mathrm{G}$ in top-heated situation ( $\mathrm{Bo}=1.20, \mathrm{Gr} \leqslant 0, \mathrm{TH})$. The corresponding first critical conditions for the onset of 3D asymmetric non-oscillatory flows and the second critical conditions for the onset of 3D oscillatory flows are determined. For the microgravity case, the axisymmetric nonoscillatory flow transfers to 3D asymmetric non-oscillatory flow with $m_{\mathrm{c} 1}=1$ at the first bifurcation and transfers to 3D oscillatory flow of (1-T) at the second bifurcation. The flow evolution in the deformed FHZ heated from bottom ( $\mathrm{Bo}=1.20, \mathrm{Gr}>0)$ is similar to the microgravity case except for the significant quantitative deviation caused by the gravity effects. On the other hand, for the flow evolution in the deformed FHZ heated from top (Bo $=1.20, \mathrm{Gr}<0)$, gravity effects alter the flow structure. Compared to the microgravity case, gravitational deformation stabilizes the axisymmetric non-oscillatory flow regardless the heating direction. However, the bottom-heated cases further stabilize the flow. On the other hand, gravity effects destabilize the 3D asymmetric non-oscillatory flow when FHZ is in top-heated situation and stabilize the 3D asymmetric non-oscillatory flow when FHZ is in bottom-heated situation. It is noted that the gravity effects on the Marangoni flow are mainly contributed by the free surface deformation. Buoyancy is less influential to the flow transitions, however, buoyancy causes multiple step transitions between different 3D asymmetric non-oscillatory flow modes.

\section{Conflict of interest}

None declared.

\section{Acknowledgements}

This research was supported by the National Natural Science Foundation of China $(11032011,11472282)$ and the Japan Society for Promotion of Science (KAKENHI 17360087).

\section{References}

[1] A. Croell, W. Mueller-Sebert, R. Nitsche, The critical Marangoni number for the onset of time-dependent convection in silicon, Mater. Res. Bull. 24 (1989) 9951004.

[2] A. Croell, P. Dolt, K.W. Benz, Segregation in Si floating-zone crystals grown under microgravity and magnetic field, J. Crystal Growth 137 (1994) 95-101.

[3] R. Rupp, G. Muller, G. Neumann, Three dimensional time dependent modeling of the Marangoni convection in zone melting configurations for GaAs, J. Crystal Growth 97 (1989) 34-41.

[4] M. Wanschura, V.M. Shevtsova, H.C. Kuhlmann, H.J. Rath, Convective instability mechanisms in thermocapillary liquid bridges, Phys. Fluids 7 (1995) 912-925.

[5] M. Levenstam, G. Amberg, Ch. Winkler, M. Andersson, Experimental and numerical studies of thermocapillary convection in a floating zone like configuration, J. Crystal Growth 158 (1996) 224-230.
[6] M. Levenstam, G. Amberg, T. Carlberg, M. Andersson, Instabilities of thermocapillary convection in a half-zone at intermediate Prandtl numbers, Phys. Fluids 13 (2001) 807-816.

[7] J.C. Chen, G.H. Chin, Linear stability analysis of thermocapillary convection in the floating zone, J. Crystal Growth 154 (1995) 98-107.

[8] M. Smith, S.H. Davis, Instabilities of dynamic thermo-capillary liquid layers. Part 1. Convective instabilities, J. Fluid Mech. 132 (1983) 119-144.

[9] R. Velten, D. Schwabe, A. Scharmann, The periodic instability of thermocapillary convection in cylindrical liquid bridges, Phys. Fluids A3 (1991) 267-279.

[10] M. Wanschura, H.C. Kuhlmann, H.J. Rath, Linear stability of two-dimensional combined buoyant-thermocapillary flow in cylindrical liquid bridges, Phys. Rev. E 55 (1997) 7036-7042.

[11] T. Hibiya, S. Nakamura, Fluid flow in silicon melt with free surface, Adv. Space Res. 24 (1999) 1225-1230.

[12] T. Hibiya, K. Nagafuchi, S. Shiratori, N. Yamane, S. Ozawa, Attempt to study Marangoni flow of low-Pr-number fluid using a liquid bridge of silver, Adv. Space Res. 41 (2008) 2107-2111.

[13] J.H. Han, Z.W Sun, L.R. Dai, J.C Xie, W.R. Hu, Experiment on the thermocapillary convection of a mercury liquid bridge in a floating half zone, J. Crystal Growth 169 (1996) 129-135.

[14] Y.K. Yang, S. Kou, Temperature oscillation in a liquid bridge and critical Marangoni number dependency on Prandtl number, J. Crystal Growth 222 (2001) 135-143.

[15] K. Takagi, M. Otaka, H. Natsui, T. Arai, S. Yoda, Z.F. Yuan, K. Mukai, S. Yasuhiro, N. Imaishi, Experimental study on transition to oscillatory thermocapillary flow in a low Prandtl number liquid bridge, J. Crystal Growth 233 (2001) 399407.

[16] S. Matsumoto, H. Hayashida, S. Yoda, A. Komiya, H. Natsui, N. Imaishi, Transition phenomena on Marangoni convection in low Pr number liquid bridge, Therm. Sci. Eng. 12 (2004) 21-22.

[17] S. Matsumoto, H. Hayashida, H. Natsui, S. Yoda, N. Imaishi, Experimental study of thermo- capillary flow in the half-zone liquid bridge of low Prandtl number fluid, JAXA Research and Development Report, JAXA, JAXA-RR-04-027E, 2005, pp. 112-128.

[18] N. Imaishi, S. Yasuhiro, Y. Akiyama, S. Yoda, Numerical simulation of oscillatory Marangoni flow in half-zone liquid bridge of low Prandtl number fluid, J. Crystal Growth 230 (2001) 164-171.

[19] S. Yasuhiro, N. Imaishi, Y. Akiyama, S. Fujino, S. Yoda, Oscillatory Marangoni flow in half-zone liquid bridge of molten tin supported between two iron rods, J. Crystal Growth 262 (2004) 631-644.

[20] S. Yasuhiro, K. Li, N. Imaishi, Y. Akiyama, H. Natsui, S. Matsumoto, S. Yoda, Oscillatory Marangoni flow in half-zone liquid bridge of molten tin, J. Crystal Growth 266 (2004) 152-159.

[21] K. Li, S. Yasuhiro, N. Imaishi, S. Yoda, Marangoni flow in half-zone liquid bridge of molten tin under ramped temperature difference, J. Crystal Growth 280 (2005) 620-631.

[22] V. Shevtsova, Thermal convection in liquid bridge with curved free surface: benchmark of numerical solution, J. Crystal Growth 280 (2005) 632-665.

[23] C. Nienhueser, H.C. Kuhlmann, Stability of thermocapillary flows in noncylindrical liquid bridges, J. Fluid Mech. 458 (2002) 35-73.

[24] H.C. Kuhlmann, C. Nienhuser, H.J. Rath, S. Yoda, Influence of the volume of liquid on the onset of three-dimensional flow in thermocapillary liquid bridges, Adv. Space Res. 29 (2002) 639-644.

[25] M. Lappa, Analysis of flow instabilities in convex and concave floating zones heated by an equatorial ring under microgravity conditions, Comput. Fluids 34 (2005) 643-770.

[26] M. Lappa, S. Yasushiro, N. Imaishi, 3D numerical simulation of on ground Marangoni flow instabilities in liquid bridges of low Prandtl number fluid, Int. J. Numer. Methods Heat Fluid Flow 13 (2003) 309-340.

[27] K. Li, B. Xun, N. Imaishi, S. Yoda, W.R. Hu, Thermocapillary flows in liquid bridges of molten tin with small aspect ratios, Int. J. Heat Fluid Flow 29 (2008) 1190-1196.

[28] K. Li, N. Imaishi, C.J. Jing, S. Yoda, Proper orthogonal decomposition of oscillatory Marangoni flow in half-zone liquid bridges of low-Pr fluids, J. Crystal Growth 307 (2007) 155-170. 